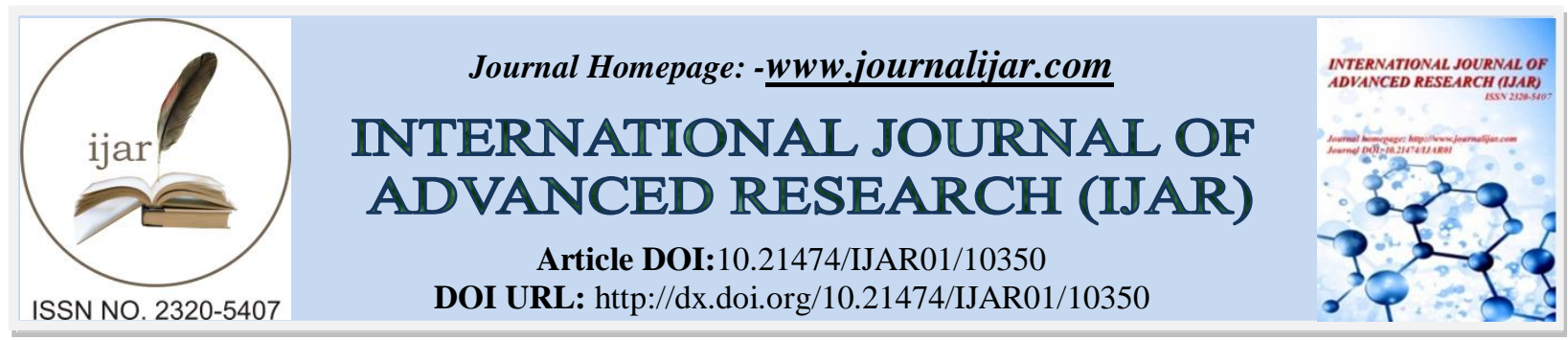

REVIEW ARTICLE

\title{
MECHANISM OF CIGARETTE SMOKE - INDUCED CHRONIC OBSTRUCTIVE PULMONARY DISEASE (COPD)
}

\section{Santanu Banerjee}

Department of Biotechnology and Dr B C Guha Centre for Genetic Engineering and Biotechnology, University College of Science and Technology, University of Calcutta, Kolkata -700019, West Bengal, India.

\section{Manuscript Info}

.1........................

Manuscript History

Received: 27 November 2019

Final Accepted: 30 December 2019

Published: January 2020

Key words:-

Chronic Obstructive Pulmonary Diseases (Copd), Cigarette Smoke (Cs), Lung, Oxidative Stress, Inflammation, Airway-Remodeling

\section{Abstract}

Chronic Obstructive Pulmonary Diseases (COPD) is currently the third leading cause of death worldwide. COPD causes great burden to society by contributing in morbidity and productivity loss. Cigarette smoking is the primary reason for COPD in smokers. In spite of the several initiatives of the national and international public health organizations for quitting smoking, number of smokers are increasing in low- and middle-income countries continuously. For the prevention and morbidity management of this treatable disease, understanding of the pathogenic events orchestrated by cigarette smoke (CS) is required. In smokers, COPD is caused by the constant exposure of lung to harmful components of CS. Oxidative stress plays the central role in CS-induced COPD pathogenesis in smokers along with other important components of COPD pathogenesis, such as lung injury, lung inflammation, protease-antiprotease imbalance, small airway remodeling and apoptosis. In this review we tried to briefly summarize the mechanisms behind the CS-induced pathogenesis for COPD which follow a complex network of events.

Copy Right, IJAR, 2020,. All rights reserved.

\section{Introduction:-}

Cigarette smoking plays a pivotal role in non-communicable diseases (NCDs) which are major contributors to the total number of global deaths. Chronic obstructive pulmonary diseases is one of the leading mortality contributor among all NCDs (Eriksen et al 2015). According to US Surgeon General Reports, cigarette smoking is the major cause of Chronic Obstructive Pulmonary Diseases (USDHHS 2004). There are more than one billion smokers in the world (Mendis et al 2011) and the number is still increasing, with the epidemic shifting to the developing world (Mendis et al 2011, Laniado-Laborín 2009). The majorities (about 80\%) of the world's smokers live in low and middle-income countries (Laniado-Laborín 2009). Global prevalence of adult tobacco smoking was almost $22 \%$ in 2010 (WHO Global status report NCDs 2014). Smoking of manufactured cigarette is the most convenient and globally practiced way of tobacco consumption (Mendis et al 2011) and used by $90 \%$ of current smokers (WHO Global status report NCDs 2014). Respiratory tract and lung are the primary organs to be exposed to cigarette smoke (CS). From lung, CS enters the bloodstream and gets access to almost every organ of the human body.

Chronic obstructive pulmonary disease (COPD) is currently the third leading cause of death worldwide (WHO: Global health estimate 2016). It was predicted that COPD will cause $7.8 \%$ of global death by 2030 (Mathers and Loncar 2006). Besides mortality, COPD attributes morbidity, productivity loss, and presents a great burden to both 
individual patients and the healthcare system. COPD was projected as the 7th leading cause of disability-adjusted life year (DALY, a WHO-recommended measure of disease burden) in 2030 (Mathers and Loncar 2006). As the most important risk factor for COPD, cigarette smoking accounts for approximately $90 \%$ of COPD cases (Peto et al 1992). Smoking causes about 80\% of all deaths from COPD (USDHHS 2014). Smokers are 12 to 13 times more likely to die from COPD than nonsmokers (USDHHS 2014).

Although the easiest way to prevent such loss from cigarette smoking is to stop cigarette smoking, it seems an unachievable tusk in present situation. In spite of several initiatives to spread the awareness about smoking related health hazards, number of smokers are increasing day by day in the middle- and low-income countries. Increase in taxes on cigarettes and forming law against smoking in public places have not change the mind of smokers so far. In this scenario prevention of cigarette smoke induced pathogenesis by molecular way is the only way to stop the harm in smokers. To achieve such goal and to develop newer and effective drug against these diseases, one must understand the pathogenic events caused by smoking at molecular level. This understanding will also help to answer the pertinent question that which specific chemical compound(s) amidst the complex mixture of cigarette CS is actually responsible for triggering the initiation of molecular reactions leading to COPD. In this review we tried to summarize the mechanisms behind the CS-induced pathogenesis for COPD which follow a complex network of events. For finding newer preventive and intervention measures, understanding of the disease pathogenesis is the primary requirement in this field of research.

\section{Pathogenesis of cigarette smoke-induced Chronic Obstructive Pulmonary Disease (COPD):}

Chronic obstructive pulmonary disease (COPD) is a common disease. It is caused by the constant exposure of lung to harmful particles and gases from environment. They induce abnormalities in alveolus and respiratory tract of lung. This ultimately leads to limitation of airflow with constant difficulties in respiration which are the basic characteristics of COPD. Timely medical intervention can prevent and treat this particular condition (Vogelmeier et al 2017). As the name suggests, COPD refers to a group of chronic pulmonary diseases such as emphysema and chronic bronchitis which cause airflow obstruction in the lung leading to troubled breathing (CDC: COPD prevention framework 2011). In emphysema, air sacs of the lung are damaged leading to enlarged airspace and breathlessness. On the other hand, chronic bronchitis is characterized by a regular cough and sputum production for at least a trimester a year for minimum two consecutive years.

Scientists tried to understand the pathogenesis of CS-induced COPD by using different suitable animal model (Churg et al 2008). Emphysematous lung damage is the major prominent phenotypic establishment in COPD patient. It was extensively studied using these models (USDHHS 2010). However, as sputum production is hard to monitor, an animal model for studying chronic bronchitis is difficult to establish (Churg et al 2008). In vivo model systems of CS-induced emphysema are used to vary in the cigarettes used, the species used, the manner in which CS is delivered, and assessment of the dose of smoke actually reaching the animals. Standard research cigarettes are generally used, and the exposure is produced by directing smoke from a single cigarette to the nose of the animal restrained in a single body compartment or by exposing groups of animals that are free to move in a chamber in which CS is put into the atmosphere (USDHHS 2010). Oxidative stress from exposure to CS has a prominent role in the pathogenesis of the COPD. Oxidative stress is also interrelated to the other components of COPD pathogenesis, such as lung injury, lung inflammation, protease-antiprotease imbalance, small airway remodeling and apoptosis (USDHHS 2010). In this review we will discuss about the specific mechanistic components of CS-induced COPD pathogenesis.

\section{CS-induced Oxidative Stress in lung:}

Exogenous oxidants from inhaled CS play the central role in oxidative stress-induced lung injury in smokers (Rahman and MacNee 1998). The lung as an environmentally exposed organ has well evolved enzymatic and nonenzymatic antioxidant systems to protect the airways and alveoli against both exogenous and endogenous oxidants. However, the balance between oxidant and antioxidant shifts unfavorably towards oxidative stress in smokers who develop COPD. The reason behind this shift is either due to the presence of an excess of oxidants or a decreased level or function of antioxidants (USDHHS 2010). In normal physiologic condition, the relative rate of production and removal of ROS is well controlled (Gutteridge 1994). Thus, in normal condition antioxidants rapidly remove the reactive oxygen species (ROS) and thereby preventing them from inducing cellular dysfunction and eventual cell death. Superoxide dismutase (SOD), catalase (Kinnula and Crapo 2003), glutathione (GSH) redox system (Rahman and MacNee 2000) and thioredoxin system (Arner and Holmgren 2000) are the major enzymatic antioxidant system of lung. 


\section{CS-induced lung epithelial injury:}

The structural integrity of the lung is contributed by the airway epithelial cells which produce mucus, defensins, matrix proteins, lipid molecules, inflammatory mediators, cell surface receptors, and antioxidants. Scientific studies observed that the epithelial cell lining of lung plays an important role in its defense from noxious foreign particles. Upon the exposure of CS-derived ROS, these cells in airway immediately response (Mercer et al 2006). In in vivo studies, it was found that CS-induced lung injury primarily initiates with increased permeability of epithelial lining ( $\mathrm{Li}$ et al 1994) leading to an increased influx of inflammatory cells in lung airway epithelial layer (Martin et al 1998).

\section{CS-induced lung inflammation:}

COPD is often characterized by the increased inflammatory response in the lungs (Hogg 2004). CS-induced oxidative stress is believed to be the responsible mechanism for the initiation and enhancement of lung inflammation which plays a major role in COPD. Lung inflammation is augmented by ROS of CS by various physiological ways, such as releasing inflammatory mediators IL-8 (Profita et al 2003), increased production of redox-sensitive transcription factors (NF- $\kappa \mathrm{B}$ and AP-1), activation of different signaling pathways such as C-JUN N-terminal kinase, extracellular signal-regulated kinase and p38 mitogen-activated protein kinase (Rahman and MacNee 1998). I- $\kappa$ B bound cytosolic inactive NF- $\kappa$ B is activated by oxidant-induced I $\kappa$ B kinase. NF- $\kappa$ B regulates the gene expression of various inflammation mediators by binding to its consensus sites in nucleus leading to inflammation. In the patients of COPD, translocation of NF- $\kappa$ B to the nucleus and its nuclear binding are increased in the airway macrophages and airway epithelial cells of the lung (Di Stefano et al 2002). As the resulting inflammation further increases oxidative stress, a harmful physiological cycle is generated where increased oxidative stress and augmented inflammation perpetuate each other (USDHHS 2010).

\section{CS-induced protease-antiprotease imbalance:}

CS-induced oxidative stress and lung inflammation both play a significant role in the process leading towards the protease-antiprotease imbalance which is considered to be the central piece of the classic theory of emphysematous lung damage (USDHHS 2010). Proteases involved in this mechanism of matrix degradation are metalloproteases, serine proteases and cysteine (USDHHS 2010). Metalloproteases, including MMP-9 and MMP-12 (Parks and Shapiro 2000), can degrade elastin (Churg et al 2008). In COPD patient, infiltrated inflammatory cells upon activation release protease. On the other hand oxidants from CS and other sources inactivate antiprotease such as AAT. These two events ultimately lead towards protease-antiprotease imbalance. In the condition of proteaseantiprotease imbalance, protease starts to degrade matrix. Some in vitro studies suggested that oxidative stress of lung in COPD patient is further increased by the protease as it induces the release of ROS from lung epithelial cells (Aoshiba et al 2001).

\section{CS-induced small airway remodeling (SAR):}

A lung tissue has repair mechanism against the potential injuries from the inhaled substances. Tissue remodeling is such a well-controlled mechanism of repair. Upon the continuous CS-induced oxidative stress and lung inflammation, the remodeling system becomes deregulated in smokers (Hogg 2004). Remodeling of small airways plays a significant role in smoker's airflow obstruction by thickening the airway walls (Hogg 2004). This tissue remodeling leads to airway fibrosis, emphysema, and pulmonary hypertension (Postma and Timens 2006). Collagen deposition is associated SAR-induced narrowing of the airway (Hogg 2004). SAR has a strong association with FEV1 (Hogg 2004).

\section{CS-induced apoptosis:}

Apoptosis of alveolar endothelial cells was emerged to be a leading phenomenon towards the initiation of emphysematous lung damage in smokers (Kasahara et al 2001). Airway lymphocytes (Majo et al 2001) and stimulated peripheral blood leukocytes (Hodge et al 2003) from patients with COPD also show increased apoptosis. There is strong evidence supporting the role of oxidative stress in apoptosis (Tuder et al 2003).

\section{References:-}

1. Aoshiba K, Yasuda K, Yasui S, Tamaoki J and Nagai A (2001) Serine proteases increase oxidative stress in lung cells. Am. J. Physiol. Lung Cell Mol. Physiol. 281(3) pp.L556-L564

2. Arner ESJ and Holmgren A (2000) Physiological functions of thioredoxin and thioredoxin reductase. Eur. J. Biochem. 267(20) pp6102-9 
3. Centers for Disease Control and Prevention (2011) Public Health Strategic Framework for COPD Prevention. Atlanta, GA: Centers for Disease Control and Prevention (Available at www.cdc.gov/copd)

4. Churg A, Cosio M and Wright JL (2008) Mechanisms of cigarette smoke-induced COPD: insights from animal models. Am. J. Physiol. Lung Cell Mol. Physiol. 294(4) pp.L612-L631

5. Di Stefano A, Caramori G, Oates T, Capelli A, Lusuardi M, Gnemmi I, Ioli F, Chung KF et al (2002) Increased expression of nuclear factor- $\mathrm{kB}$ in bronchial biopsies from smokers and patients with COPD. Eur. Respir. J. 20(3) pp556-63

6. Eriksen M, Mackay J, Schluger N, Gomeshtapeh F and Drope J (2015) The Tobacco Atlas. 5ed: Revised, Expanded, and Updated (Atlanta, USA)

7. Gutteridge JM (1994) Biological origin of free radicals, and mechanisms of antioxidant protection. Chem. Biol. Interact. 91(2) pp133-140

8. Hodge SJ, Hodge GL, Reynolds PN, Scicchitano R and Holmes M (2003) Increased production of TGF- $\beta$ and apoptosis of T lymphocytes isolated from peripheral blood in COPD. Am. J. Physiol. Lung Cell Mol. Physiol. 285(2) pp.L492-L499

9. Hogg JC (2004) Pathophysiology of airflow limitation in chronic obstructive pulmonary disease. Lancet 364(9435) pp709-21

10. Kasahara Y, Tuder RM, Cool CD, Lynch DA, Flores SC and Voelkel NF (2001) Endothelial cell death and decreased expression of vascular endothelial growth factor and vascular endothelial growth factor receptor 2 in emphysema. Am. J. Respir. Crit. Care Med.163(3) pp.737-744

11. Kinnula VL and Crapo JD (2003) Superoxide dismutases in the lung and human lung diseases Am. J. Respir. Crit. Care Med. 167(12), pp.1600-1619

12. Laniado-Laborín R (2009) Smoking and chronic obstructive pulmonary disease (COPD). Parallel epidemics of the 21st century. Int. J. Environ. Res. Public Health 6(1) pp.209-224

13. Li XY, Donaldson K, Rahman I and MacNee W (1994) An investigation of the role of glutathione in increased epithelial permeability induced by cigarette smoke in vivo and in vitro. Am. J. Respir. Crit. Care Med. 149(6) pp1518-25

14. Majo J, Ghezzo H and Cosio MG (2001) Lymphocyte population and apoptosis in the lungs of smokers and their relation to emphysema. Eur. Respir. J. 17(5) pp.946-953

15. Martin LD, Krunkosky TM, Voynow JA and Adler KB (1998) The role of reactive oxygen and nitrogen species in airway epithelial gene expression. Environ. Health. Perspect. 106 Suppl 5 pp1197-1203

16. Mathers CD and Loncar D (2006) Projections of global mortality and burden of disease from 2002 to 2030. PLoS. Med. 3(11): e442

17. Mendis S, Puska P, Norrving B and World Health Organization (2011) Global atlas on cardiovascular disease prevention and control Geneva: World Health Organization

18. Mercer BA, Lemaitre V, Powell CA and D'Armiento J (2006) The epithelial cell in lung health and emphysema pathogenesis. Curr. Resp. Med. Rev. 2(2) pp101-142

19. Parks WC and Shapiro (2000) Matrix metalloproteinases in lung biology. Respir. Res.2(1) pp.10-19

20. Peto R, Lopez AD, Boreham J, Thun M, and Heatch C (1992) Mortality from tobacco in developed countries: indirect estimation from national vital statistics. Lancet 339(8804) pp1268-1278

21. Postma DS and Timens W (2006) Remodeling in asthma and chronic obstructive pulmonary disease. Proc. Am. Thorac. Soc. 3(5) pp.434-439

22. Profita M, Chiappara G, Mirabella F, Di Giorgi R, Chimenti L, Costanzo G, Riccobono L, Bellia V et al (2003) Effect of cilomilast (Ariflo) on TNF- $\alpha$, IL-8, and GM-CSF release by airway cells of patients with COPD. Thorax 58(7) pp573-9

23. Rahman I and MacNee W (1998) Role of transcription factors in inflammatory lung diseases. Thorax 53(7) pp 601-612

24. Rahman I and MacNee W (2000) Regulation of redox glutathione levels and gene transcription in lung inflammation: therapeutic approaches. Free Radic. Biol. Med. 28(9) pp1405-1420

25. Tuder RM, Zhen L, Cho CY, Taraseviciene-Stewart L, Kasahara Y, Salvemini D, Voelkel NF and Flores SC (2003) Oxidative stress and apoptosis interact and cause emphysema due to vascular endothelial growth factor receptor blockade. Am. J. Respir. Cell Mol. Biol. 29(1), pp 88-97

26. U.S. Department of Health and Human Services (2010) How Tobacco Smoke Causes Disease: The Biology and Behavioral Basis for Smoking-Attributable Disease: A Report of the Surgeon General. Office on Smoking and Health Atlanta (USA) 
27. U.S. Department of Health and Human Services (2014) The Health Consequences of smoking- 50 years of progress; A report of the Surgeon General. Department of Health and Human Services. Public Health Service. US Office of the Surgeon General

28. US Department of Health and Human Services (1983) The Health Consequences of Smoking: Cardiovascular Disease. A Report of the Surgeon General. Rockville, MD, US Department of Health and Human Services. Public Health Service, Office on Smoking and Health.

29. US Department of Health and Human Services (2004) The health consequences of smoking: a report of the Surgeon General Office of the Surgeon General (US)

30. Vogelmeier CF, Criner GJ, Martinez FJ, Anzueto A, Barnes PJ, Bourbeau J, Celli BR, Chen R et al (2017) Global strategy for the diagnosis, management, and prevention of chronic obstructive lung disease 2017 report. GOLD executive summary. Am. J. Respir. Crit. Care Med. 195(5) pp557-582

31. World Health Organization (2018) Global Health Estimates 2016: Deaths by Cause, Age, Sex, by Country and by Region, 2000-2016. Geneva, World Health Organization. 